${ }_{24}^{4} / 20$

$(1)$

\section{4,3990}

UCID- 78876

\title{
Line Shapes for Some Specific Transitions in Hydrogenic, Lithium-Like, and Helium- \\ Like Ions
}

\section{MASTER}

R. W. Lee

December 29, 1980

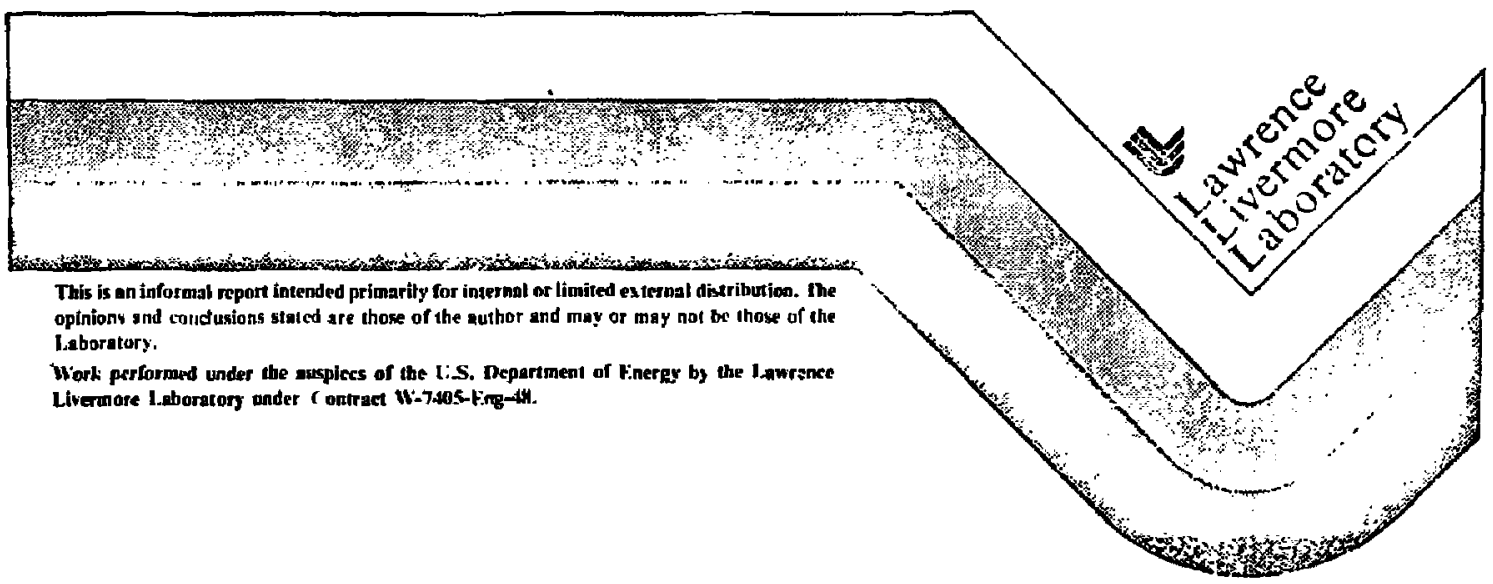


Line Shapes for Some Specific Transitions in Hydrogenic, Lithium-Like, and Helium-

Like Ions

by

R. W. Lee**, Imperial College, London

University of California, Lawrence Livermore National Laboratory

P.0. Box 5508, Livermore, California 94550

\section{ABSTRACT}

A set of computer codes have been developed to produce spectral line shapes of highly stripped lons for a number of transitions in Hydrogenic, Helium-like and Lithium-1ike species. The choice of the transitions has been dictated largely by the laser compression experiments. The transitions of Hydrogenic species are: Lyman $\alpha$; Lyman $\beta$; Lyman $Y ; L y m a n \delta$;

Balmer $\alpha$; and Balmer $\beta$. The transitions of Helium-?ike species are: $1 s^{2}\left({ }^{1} s_{0}\right)-1 s 2 p\left({ }^{1} P\right) ; 1 s^{2}\left({ }^{1} s_{0}\right)-1 s 3 p\left({ }^{1} P\right) ; 1 s^{2}\left({ }^{1} s_{0}\right)-1 s 4 p\left({ }^{1} P\right)$ and the associated dipole-forbidden components. The transitions of Lithium-like species are the entire $n=2$ to $n=3$ and $n=4$ complexes; ten transitions are calculated: $1 s^{2} 2 s^{2} s_{1 / 2}-1 s^{2} 3 p{ }^{2} p_{1 / 2,3 / 2} ; 1 s^{2} 2 p^{2} p_{3 / 2}-1 s^{2} 3 s^{2} s_{1 / 2}$; $1 s^{2} 2 p^{2} p_{3 / 2}-1 s^{2} 3 d^{2} 0_{3 / 2,5 / 2} ; 1 s^{2} 2 p{ }^{2} p_{1 / 2}-1 s^{2} 3 s^{2} s_{1 / 2}$; $7 s^{2} 2 p^{2} P_{1 / 2}-7 s^{2} 3 d^{2} D_{3 / 2,5 / 2}$;

and the same transitions, for level $n=4$. A companion note will describe a line shape fitting program which can be used together with these profiles and experimental data.

* Consultant to Y-Program at LLNL. Permanent Address: Spectroscopy Blackett Laboratory, Imperial College, London England

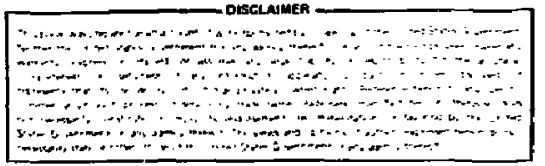




\section{Introduction}

The calculation of line shape requires sufficient specialized knowledge to deflect most individuals from attempting to obtain line shapes for the particular purposes in which they are interested ${ }^{l}$. These purposes could be the calculation of opacities, the fitting of spectral line shapes to obtain information about plasma parampters and/or studying the line shape itself as a means of understanding the detailed coupling of the plasma to the atom. The motivation here is to provide an easy method to obtain spectral line profiles for a small subset of interesting transitions. The transitions chosen are primarily of interest in the diagnosis of high density laser compression (usually exploding pusher type) experiments. For example, a possible diagnostic method is simply to determine the electron temperature and plasma electron density of interest, the mean ion charge surrounding the $x$-ray emitter; then decide which of the element's last 3 ion stages are relevant, run the code, ànd obtain a "fit" between theory and experiment.

it should be noted that the calculation of the line profile is somewhat time-consuming. At the time of writing, the code uses about 45 seconds for the hydrogenic species, 30 seconds for Helium-like species and 90 seconds for the Lithium-like lines. 


\section{Theoretfcal Background}

\section{a) Energy Levels}

The calculated line profiles require a knowledge of the energy levels and oscillator strengths. In the case of Hydrogenic species the simple hydrogen-like atom approximation is used which has degenerate levels in all states. However, since the fine structure splitting of the $n=2$ level is $-5 m \AA$ (for all hydrogenic ions), the effect is included for the Lyman $\alpha$ transition. The data for the Helium-like levels and oscillator strengths was calculated by Dr. G. Bromage ${ }^{2}$ using the Cowan-Zealot code at Astrophysical Research Division, Rutherford Laboratory, England. However, the Lithium-like energy levels and oscillator strengths for the case of Bromine were calculated by J. Scofield of T division (LLNL). The data for Helium-like and Lithium-like species are spotty so that data on a particular atomic species may have to be input (by the user) into the code's appropriate common blocks.

b) The Microfields

The ion quasi-static microfields are calculated within the code using a method devised by A. G. Richards based on formulation of the microfield given by Hooper ${ }^{3}$. The microfield calculations (and indeed all basic aspects of the spectral line broadening problem) are discussed in 
"Spectral Line Broadening in PJasmas" by H. Griem"

\section{c) The Collision Operators}

The effects of dynamic collision must also be included, and this is achieved with the modified relaxation theory method ${ }^{1}$. The method as applied here truncates the interaction after second-order in the plasma-ions interaction potential. In this way the line cores are calculated quite accurately, but the wings of the line, i.e., the region where the quasi-static perturbations of the line dominate, will not be we11 represented. However, given the observability of the line wings from a highiy stripped ionic emitter, this is no great loss for those who want to use line profiles as a diagnostic, where one looks at the core region of the line, or for those interested in the details of plasma features reflected in the line shape. Note, for the calculation of opacity this is a serious drawback since the Rosseland Mean Opacity is a harmonic mean, and, thus, gives highest weighting to those spectral regions where the opacity is lowest. This region of low opacity is usually synonymous with the line wings. Therefore, for opacity calculations, the line wings will have to be viewed with great care!

In order to calculate the collision operators, we employ the quantum mechanical Random phase dielectric function. This allows one to study the plasma correlations, in a equilibrium plasma, as they affect a line 
profile. The second order collision operator formulation has been supplemented with a "strong collision" term to account for the fact that the second order expression will not correctly treat the strong collisions. This aiso requires a strong collision cut-off which can be derived as a minimum impact parameter allowed for a plasma perturber. The two posstble choices are derived by imposing unitarity of the approximated t-matrix or requiring that the perturber not intercede between the transition electron and the nucleus.

d) The Line Profile

Schematically, the line shape is calculated as a convolution of two separate types of broadening mechanisms. A quasi-static microfield profile $P(E)$ (similar to a Holtsmark or nearest-neighbor shape) and a dynamic profile (similar in form to a Lorentzian type) $\phi(\omega, E)$. Thus, to get the final line profile $\$$ we have

$$
\Phi_{(w)}=\int d E P_{(E)} \phi_{(W, E)}
$$

where $E$ is the microfield strength. The precise form of complex quantity, can be studied by reference to H. Griem's book. However, in a greatly simplified manner we have

$$
\phi_{(W, E)}=\operatorname{Im}(\Delta W(E)+i H(M, s))^{-1}
$$




$$
-6-
$$

where $w(E)$ is the unperturbed energy difference of the levels in the Stark representation, thereby, becoming a function of field strength $E$. and $H(W, E)$ is the collision operator which contains both real and imaginary parts, which are often called in the literature, the shift and width operators, respectively. For the final output, the line profile is convolved with the appropriate Doppler profile so that Doppler motion is included in the output spectral profile.

\section{Validity}

The range of validity for these calculations varies with ion stage. First the Hydrogenic and Helium-like profile have been extensively used in both high and low density plasmas, and tine results are satisfactory. (See discussions contained in Reference 2.) Tie errurs est imated are, at worst, about $50 \%$ (which as far as density diagnostics are concerned is not too bad since the temperature and density region where the shapes will be most unreliable is where the plasma is very fense and relatively cool). In these regions; the lines overlap, introducing new levels into the problem, and the plasma formulation, which requires at least one particle per Debye sphere, actually breaks down.

The case of the Lithium-like structures is different since theoretical formulation was actually performed just prior to the writing of this memo. So far it has been evaluated only for Li-like Bromine following 
one laser compression experiment. The required calculation of the energy levels becomes more critical in cases Tike BR XXXIII because the allowed transitions start to merge. Then it is difficult to ascribe oscillator strengths to unresolved features. In this case, Li-like atomic level data will have to be included in order to run the program.

A bit of advice: the program, unlike the remainder of the viorld, is not constrained to denave accoroting to the laws or physics. In generap, the output line profiles should appear relatively smooth, the sharfest features should be the resonance features. However, when there is an adjacent electric dipole-forbidden t:-3nsition, the wing of the allowed line transition nearest the forbidden line transition may decrease much more rapidly than an opposing allowed Tine wing.

\section{Running the Code}

\section{A Inputs}

Once the correct code is obtained, see the appendixes for details of the storage and modification of the code; one only has to specify five quant it ies: 
Density of electrons in number $/ \mathrm{cm}^{3}: \mathrm{N}_{\mathrm{e}}$

Atomic number of the element of interest: $Z_{A}$

Charge of the surrounding ions: 2

Number of plasma species: $\operatorname{KIND}(=1)$

\section{B. Example of Input}

Assuming we obtain the controlee "RUNHE" which will run Helium-like transitions, and assuming we wish to run the case of He-like Argon lines emitted from a plasma with temperature of $1 \mathrm{KeV}$, an electron of $10^{24} \mathrm{e}^{-} / \mathrm{cm}^{3}$ and an abundance of D-T with negligible Argon (i.e., an Argon seeded D-T fili). Then following are the necessary statements: (where the code responds with a "?" where it wants input statements)

RUNHE / T V

? TEMP

? $1.1605 \mathrm{E}+07$

? DENS

? $1.00 \mathrm{E}+24$

? ZEDS
Run the ccntrolee Indicates next variable is $T$. Note the end of an exponent field must be in column 10. Also note this is ${ }^{\circ} \mathrm{K}$. Indicates next variable is $\mathrm{N}_{\mathrm{E}}$ Again exponent must be in column 10 Indicate next input will be the 
$?$

18. 2.

3 KIND

$?$ ?

? 18.
Atomic Number of Argon, and the mean ion change is estimated at 2 . Note that the 2. should start after column 19.

Indicates next input is number of plasma constituents which will effect the collisional broadening.

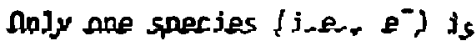
chosen. Note the 1 must be in calumn 2.

Plasma electrons only Input Zed The code confirms that on ly one species will be run and asks for reconfirmation of atomic number of emitter.

Reply with Atomic Number of Argon.

From the time of the last input, i.e., the final confirmation of the Atgon atomic number, the coute witi rum the ifine proficies, and a mesponse will only be given when it is finished generating the line profiles. Before we discuss the final output, a few coments on the input are necessary. First, at any time until the word "KIND" is entered, the other variables can be rewritten by repetition of the input line indicator, such as "DENS" to input the density again-(the last entry will be accepted). 
The major reason to have the input in this way is to keep this program compatible with one which is operating at Imperial College.

\section{Output}

\section{Diagnostic Printout: TAPE7}

The output will be in two forms. One, a line printer formatted listing of the inputs, microfields, collision operators and line shapes will be produced on tile TAPE7. This can be viewed easily using either TRIX AC with the long line option or with TUBE with the "small" option. This "print" file is actually rict intended for any purposes but diagnostics of bad results. However, it can be interesting if one wants to understand the profiles in more detail. A description of the file TAPE7 is given in Table 1.

\section{Binary File With Usable Information TAPEI}

Second, the useful output is a binary file, which contains all the relevant data required to use the line shape in a companion program which does fitting to experimental data. The fact that it is a binary file and that it is generated using an FTN code means that to use it in a CHAT code, one must use fTNCONV. Thus, the output binary which is always 
TAPE 1 can be converted to CHAT usable file with following statement

\section{FTMCONV TAPEI TAFECHT / T V}

where TAPECHT is now a CHAT compatible binary file. In Table 2, tise contents of TAPEI are shom. Note that most of the information is not necessary for running a fitcing routine or finding an opacity; however, it has been retained so that restarts could be made possible with a minimum of effort. In Table 4 list of the line profiles and the order in which they are written on TAPEI is given.

This constitutes the output. 
Table 1

TAPE] File

ITEM 1

ITEM 2

ITEM 3

ITEM 4

ITEM 5
Information about inputs and microfield variables.

Line printer plot of microfield probability distribution vs mean field strength $E_{0}$. Information on the calculation of the collision operator. But, the form here is raw data.

Information on calculations. This is the shift and width operators for various cases;essentially raw data.

Line printer plots of line shape vs energy (eV) for the profile A) without Doppler broadening $B$ ) with Doppler broadening. That is, two plots for each transition 
Tabte 2

Contents of Binary File TAPEI

Record

1

2

3

4

\section{Contents}

Message 4 character per word blocks

Temperature $\left({ }^{\circ} \mathrm{K}\right)$; electron density ( $\# / C c)$; atomic iumber of emitter charge of plasma mean ion; charge of second mean ion species; (here set to zera) number of plasma constituents $(K[N D=1)$.

Ratios of the number density, charge, mass, temperature, and drift velocity; for "KINO" number of species relative to the plasma electron values (in this case KIND $=1$ implies that all these numbers are 1$)$.

Information in microfield spacing $N 1, N 2,01, D 2$. Where $N 1$ is the number of field points with mean field increment $\mathrm{Dl}$; and N2-MI is the number of points with mean field increment 02 . 
Microfield probabijity distribution

$P(E)$ for $N 2$ points in total.

6

NWAVE = number of frequency points

in the data for the collision

operators.

7

The NWAVE frequencies for the

collision operators.

8

$\left(H(I, J)_{\text {real, }} H(I, J)_{\text {imag inary, }} I=1\right.$, NWAVE $)$, $\mathbf{J}=1$, \# of energy levels of interest). (These are the broadening operators proper). \#= 5 for hydrogenic $\#=10$ for He-like, \#= 7 for Li-like

((AW $(I, J) .1=103) \mathrm{J}=1$, NUM), ((WAVE $(I, J), I-1,(103), J=1$, NUM), (ZLAM( 1), DUM, DUM, I = 1, NUM) where NUM is the number of line profile: 5 for bydrogenic, 3 for tie- i ike, 10 for Li-like. The At are 
code internal energy units; WAVE are the energy units in EV; ZLAM is the transition center energy EY.

12 through $12+$ NUM-1*

$(\operatorname{PHI}(1, J), I=1,103), J=1,3)$

The line profiles for the three cases:

1. Profile for the case of one microfield (i.e., unaveraged) with a value of $5 E_{0}$.

2. Profiles for all effects but no Doppler convolution.

3. Profile for all effects, including Doppler and normalized so that total area equals to oscifluator strength of the transition (Hente, directly usable in the fitting of exper imental data).

*See Table 4 for the order of the line profiles in the various cases. 
Table 3

Overlay Structure

Over lay

Function

0,0

Main:

Calís a il other over iays.

1,0

Dato Input:

Initialize datd with

interactive inputs. Create

Tape 1.

2,0

Microfield:

Reads records 1 through 3 of

Tape 1, creates microfield,

writes records 4 and 5 on

Tape 1.

3,0

Collision

Operators:

Reads records I througi 3 of

TAPE 1, creates collision

operators, writes records 6

through 10 on Tape 1.

4,0 Line Shapes:

Reads records 1 through 10 , creates line profile, writes records 11 through $12+k i v i 4+i^{*}$.

* See Table 2. 
Table 4

Line Profile Assignments

\section{TRANSITION}

$1=$ Lyman $\alpha$

$2=L y \operatorname{man} B$

$3=$ Lyman $r$

$4=\operatorname{Lyman} \delta$

$5=\operatorname{Balmer} \alpha$

6 = Balmer $B$

$I=1 s^{2}{ }^{1} S_{0}-I s 2 p{ }^{1} p$

$2=1 s^{2} 1_{s_{0}-1 s 3 p}{ }^{1} p$

$3=1 s^{2} l_{s_{0}-1 s 4 p} l_{p}$

\section{ELEMENT}

$1=$ Hydrogen ic

$2=$ Hel ium-Like 
$1=2 S-3 P$

$2=2 P_{3 / 2}-35$

$3=2 P_{3 / 2}-30$

$4=291 / 2^{-35}$

$5=2 P_{1 / 2^{-3 D}}$

$3=$ Lith ium-Like

$5=2 S-4 P$

$7=2 P_{3 / 2}-4 S$

$8=2 P_{3 / 2}-4 D$

$9=2 p_{1 / 2^{-45}}$

$10=2 P_{1 / 2}-40$ 
Li-Like - Oscillator Strength for Transition Name

$$
\begin{array}{lr}
2^{2} S_{1 / 2}-3^{2} P_{1 / 2,3 / 2} & \text { OS1 } \\
2^{2} P_{3 / 2}-3^{2} S_{1 / 2} & \text { OS2 } \\
2^{2} P_{3 / 2}-3^{2} D_{3 / 2,5 / 2} & \text { OS3 } \\
2^{2} P_{1 / 2}-3^{2} S_{1 / 2} & \text { OS4 } \\
2^{2} P_{1 / 2}-3^{2} D_{3 / 2} &
\end{array}
$$

$2^{2} S_{1 / 2}-4^{2} P_{1 / 2,3 / 2}$

$0 S 6$

$2^{2} p_{3 / 2}-4^{2} s_{1 / 2}$

0S7

$2^{2} P_{3 / 2}-4^{2} D_{3 / 2,5 / 2}$

OSB

$2^{2} p_{1 / 2}-4^{2} S_{1 / 2}$

DSy

$2^{2} P_{1 / 2}-4^{2} D_{3 / 2}$

Li-Like data exist for atomic numbers $Z=3,22,35$ 
Table 5

Data For Line Shapes

He-Like

\begin{tabular}{|c|c|c|}
\hline $\begin{array}{l}\text { Energy of Leve } 1 \\
1 s^{2} s^{1} s\end{array}$ & $\begin{array}{l}\text { Variable Name } \\
\text { W2S }\end{array}$ & $\begin{array}{l}\text { Units } \\
\mathrm{CM}^{-1} \\
\text { (relative to } \\
\left.1 \mathrm{~s}^{2} \mathrm{l}_{\mathrm{S}_{0}}\right)\end{array}$ \\
\hline $1 s 2 p^{1} p$ & W2P & " \\
\hline $1 s 3 s^{1} s$ & H3S & $"$ \\
\hline $1 s 3 p^{1} p$ & W3P & " \\
\hline $1 \mathrm{~s} 3 \mathrm{~d}^{1} \mathrm{D}$ & W30 & " \\
\hline $1 s 4 s^{1} s$ & W4S & " \\
\hline$I s 4 p^{1} p$ & W4P & " \\
\hline $1 s 4 d^{1} D$ & WAD & " \\
\hline $1 s 4 f^{l} F$ & WAF & " \\
\hline
\end{tabular}




$$
-21-
$$

He-Like Oscillator Strength of Transition Variable Name

$$
\begin{array}{ll}
1^{1} S-2^{1 p} & \text { OS1 } \\
1^{1} S-3^{1} p & \text { OS2 } \\
1^{1} S-4^{1} p & \text { 0S3 }
\end{array}
$$

He-Like data exist for atomic numbers $Z=2,4,6,8,10,13,14,18$ 
Table 6

Data for Line Shapes

\begin{tabular}{|c|c|c|}
\hline $\begin{array}{l}\text { Li-Like - Energy of Level } \\
\qquad 1 s^{2} 2 p^{2} P_{1 / 2}\end{array}$ & $\begin{array}{l}\text { Name } \\
\text { W2P12 }\end{array}$ & $\begin{array}{l}\text { Units } \\
\text { Ev (relative to } \\
\left.1 s^{2} 2 s\left(s_{1 / 2}^{2}\right)\right)\end{array}$ \\
\hline $1 s^{2} 2 p^{2} p_{3 / 2}$ & $12 P 32$ & $m$ \\
\hline $1 s^{2} 3 s^{2} s_{1 / 2}$ & W3S12 & $"$ \\
\hline $1 s^{2} 3 p^{2} p_{1 / 2}$ & W3P12 & $"$ \\
\hline $1 s^{2} 3 p^{2} P_{3 / 2}$ & W3P 32 & $"$ \\
\hline $1 s^{2} 3 d^{2} 0_{3 / 2}$ & W3D32 & $"$ \\
\hline $1 s^{2} 3 d^{2} D_{5 / 2}$ & W3052 & $"$ \\
\hline $1 s^{2} 4 s^{2} s_{1 / 2}$ & W4S12 & " \\
\hline $1 s^{2} 4 p^{2} p_{1 / 2}$ & WAP12 & " \\
\hline $1 s^{2} 4 p^{2} P_{3 / 2}$ & WAP32 & " \\
\hline
\end{tabular}


$-23-$

$1 s^{2} 4 d^{2} D_{3 / 2}$

W4032

$1 s^{2} 4 d^{2} D_{5 / 2}$

W4D52

It

$1 s^{2} 4 f^{2} F_{5 / 2}$

W4F52

$\omega$

$1 s^{2} 4 f^{2} F_{3 / 2}$

W4:72

II 
Appendix

\section{A. Structure of the Code}

\section{General Corments}

The codes are written in standard FTN type fortran and are compatible with the computers at Imperial College. This method is kept so that any "glitches" or "bugs" can be reprofuced at IC. This, however, means that the FTN facilities must be used. Note that the two major differences are the overlaying structure and the binary read/write characteristics.

\section{Code Structure}

The code is overlayed with the main and four p' mary overlays. The main overlay only calls the remaining four overlays in orfer to control the operation of the code. Table 3 indicates the function of each overlay.

B. Where to Find Code

1. The controlees RUNH, RUNHE, RUNLI are kept on Dennis Mat thews" (Y Division $\times 25360$ ) number in a fhotostore directory, i.e., 
2. If it becomes fiecessary to add to the data set, to calculate atomic elements not contained in the codes or just modify the source, the sources are called (imaginatively) Hydrogen, Helium, Lithium.

\section{How to Run FTN Over layed Code}

In order to run an FTH over layed code, one not only needs the code but also an overlay structure file. These files are also kept in the photostore and are called OVLAYH, OVLAYHE, OVLAYLI.

A two step compilation has been used, although one step is probably sufficient. That: is when Hydrogen, Helium and Lithium, and OVLAYX (where $x$ is $H$, He or LI) has been obtained and modified, the following steps produce a controllee RUNX.

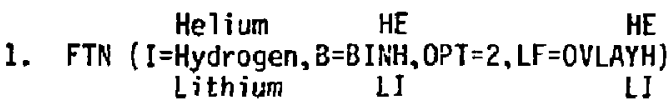

2. HE HE HE

2. FTN (CNAME=RUNH, LDONLY, GLIB $=B$ INSH, LF=OVLAYH)

$$
\text { LI LI LI }
$$

D. Present Data Base

The data base required for the Lithium-like and Helium-like are the energy levels and the oscillator strengths. The data is stored in each 
programe in "BLOCK DATA BDO" in data statements. Table 5 shows the variable, its local code name, units of the variable and which data exist.

Note carefully, that although output. of all the codes is in EV, the energy level information for the Helium-like species is in $\mathrm{cm}^{-1}$, where $1.2398 \times 10^{-4} \mathrm{eV}-\mathrm{cm}$ ! Further, the arrays for Helium are present $1 \mathrm{y}$ dimensioned at 18 implying the Argon is the highest $Z$ species possible without redimensioning, while for Lithium the highest is $Z=36$ or Krypton without redimensioning.

E. Summary for Running Codes

Files: RUNH, RUNHE, RUNLI

Stored: $\quad .575314:$ RhLEE

Purpose: Calculate Some Hydrogenic, Helium-like, Lithium-like Line Profiles

Inputs: Plasma Temperature, Electron Density, Mean Plasma Ion Charge, Emitter Atomic Number (Section 4A)

Procedure: Sée Section 4b

Outputs: See Section $4 c$ 


\section{REFERENCES:}

1. H. Griem "Spectral Line Brcadening in Plasmas" (Acadenic Press, New York; 1974)

2. a) R. W. Lee, J. Phys B: 121112 (1979)

b) R. W. Lee, J. Phys B: 12 1129 (1979)

c) R. H. Lee, J. Phys B: 121144 (1979)

d) R. W. Lee, G. E. Bromagz and A. G. Richards, J. Phys B: $123445(1979)$

e) R. W. Lee and A. J. Freeman, JQSRT, 24, 43 (1980)

3. J. O'Brien and C. F. Hooper, Phys Rev A5, 867, (1972) 